OPEN

SUBJECT AREAS:

CANCER IMAGING

AUTOIMMUNITY

Received

4 July 2013

Accepted

27 March 2014

Published

30 May 2014

Correspondence and requests for materials should be addressed to

S.M.S. (simon@mail. rockefeller.edu)

* These authors contributed equally to this work.

\title{
Endogenous Antibodies for Tumor Detection
}

Barrie S. Rich',2*, Joshua N. Honeyman 1,2*, David G. Darcy 1,2, Peter T. Smith', Andrew R. Williams', Irene Isabel P. Lim, ${ }^{1,2}$ Linda K. Johnson ${ }^{3}$, Mithat Gönen ${ }^{4}$, Joel S. Simon ${ }^{5}$, Michael P. LaQuaglia ${ }^{2}$ \& Sanford M. Simon'

'Laboratory of Cellular Biophysics, The Rockefeller University, New York, NY 10065, ${ }^{2}$ Division of Pediatric Surgery, Department of Surgery, Memorial Sloan-Kettering Cancer Center, New York, NY 10065, ${ }^{3}$ Center of Comparative Medicine and Pathology, Laboratory of Comparative Pathology, Memorial Sloan-Kettering Cancer Center, New York, NY 10065, ${ }^{2}$ Department of Epidemiology and Biostatistics, Memorial Sloan-Kettering Cancer Center, New York, NY 10065, ${ }^{5}$ The Dalton School, New York, NY 10128.

The study of cancer immunology has provided diagnostic and therapeutic instruments through serum autoantibody biomarkers and exogenous monoclonal antibodies. While some endogenous antibodies are found within or surrounding transformed tissue, the extent to which this exists has not been entirely characterized. We find that in transgenic and xenograft mouse models of cancer, endogenous gamma immunoglobulin (IgG) is present at higher concentration in malignantly transformed organs compared to non-transformed organs in the same mouse or organs of cognate wild-type mice. The enrichment of endogenous antibodies within the malignant tissue provides a potential means of identifying and tracking malignant cells in vivo as they mutate and diversify. Exploiting these antibodies for diagnostic and therapeutic purposes is possible through the use of agents that bind endogenous antibodies.

(1) here are a multitude of different types of cancer, and within a specific tumor type there is considerable patient-to-patient and intra-patient variability, reflecting the fact that cancer is a dynamic and heterogeneous collection of diseases ${ }^{1,2}$. Each individual tumor has a distinct set of mutations resulting in different molecular profiles ${ }^{3,4}$. Tumors continue to evolve, particularly when selective pressures are applied, such as chemotherapy and radiation. As a result, any recurrence or metastasis may have a very different genetic profile from the primary tumor. This variability has been implicated in diagnostic complexity and resistance to therapy ${ }^{5,6}$.

While it is accepted that that the immune system can recognize tumors, there are conflicting data as to whether the immune system can suppress tumors. By one hypothesis, tumors can elicit an immune response within the host, but it is not sufficient to prevent tumor progression ${ }^{7}$. The immunoediting hypothesis posits that somatic cells are constantly acquiring mutations that lead to malignant transformation, and the immune system is able to eliminate these developing tumors. Cancer is a consequence of the immune system being insufficient to prevent tumor progression. This is consistent with the observation that immunosuppressed transplant recipients have higher rates of viral and non-viral associated tumors than the general population ${ }^{8-11}$. Cancer becomes a clinical problem when the malignant cells acquire the necessary mutations to evade cellular regulatory mechanisms, escape elimination by the immune system, and invade the surrounding tissues ${ }^{12}$. The tumors are imprinted by the immunologic environment in which they develop, and selective pressure yields tumors that are better able to evade the immune system. Regardless of how the immune system fails to completely suppress tumors, both hypotheses recognize that there is immunological recognition of the transformed cells.

In clinical oncology, the host immune response is used in various ways for both diagnosis and treatment. Humoral-based immunotherapy primarily uses exogenous monoclonal antibodies ${ }^{13}$. There are monoclonal antibodies undergoing extensive investigation that target numerous cell-surface proteins, antigens in tumor stroma, antigens on tumor-associated vessels and ligands that maintain tumor growth. Many therapeutic antibodies, including trastuzumab, bevacizumab, and cetuximab, improve outcomes in various tumor types and are clinically approved for therapy ${ }^{13}$.

Endogenous antibodies generated in response to aberrant proteins associated with tumors may be found in patient serum. A number of tumor-associated autoantibodies have been identified circulating in the serum in the hopes of utilizing them as biomarkers, prognostic factors, or indicators of tumor recurrence ${ }^{13}$. For example, 
autoantibodies to NY-ESO-1 have been identified in the serum of patients with esophageal, lung, liver, breast, thyroid, prostate, and colorectal cancers ${ }^{14-19}$. In patients with hepatocellular carcinoma (HCC), probing for a single autoantibody in the serum gives a positive result in $10-20 \%$ of patients; the detection increases to $66 \%$ with a panel of ten autoantibodies ${ }^{20}$. While the sensitivity of tumor detection can be increased by using a panel of antibodies over a single antibody ${ }^{21}$, the results are still insufficient for diagnosis in many tumor types. If a panel of ten antibodies is more sensitive than a single antibody, the entire repertoire of a patient's antibodies might be even more effective at detecting tumors. Endogenous antibodies have also been observed within, and adjacent to, neoplastic tissue. IgG has been demonstrated within soft tissue sarcomas and papillary thyroid cancer ${ }^{22,23}$. In soft tissue tumors its presence was associated with proliferation markers and increasing tumor grades ${ }^{22}$.

This project is designed to test if the spectrum of an individual's antibodies found within tissue can be used to differentiate neoplastic from normal tissue, which opens the potential for use in cancer detection and localization. In one xenograft and three transgenic mouse models of cancer, we find that endogenous antibodies reliably bind neoplastic tissue and distinguish it from wild-type tissue. The endogenous antibodies within the tumor recognize a diversity of transformed cells. This humoral immune response co-evolves with the malignant cells and may represent a target for diagnosis and therapy.

\section{Methods}

Mouse models. The Alb/c-myc mouse models ${ }^{24}$ were a generous gift from Herman Stellar and both prostate models (PB- $M y c^{25}$ in FBV background and conditional $\mathrm{PTEN}$ knockout $\left(\mathrm{Pb}-\mathrm{Cre} \times \mathrm{Pten}^{\mathrm{f} / \mathrm{f}}\right)$ in $\mathrm{C} 57 / \mathrm{B} 6$ background $\left.{ }^{26}\right)$ were a generous gift from Charles Sawyers. Alb/c-myc and prostate sample controls were C57BL/6J mice purchased from The Jackson Laboratory. All FVB/N-Tg(MMTVneu)202Mul/J mice, and the corresponding control FVB/NJ mice were purchased from The Jackson Laboratory. In the xenograft model we injected six-week-old BALB/c mice (Jackson Labs) and CBySmn.CB17-Prkdc scid/J mice (Jackson Labs) with a 4T1 cell line. Five thousand cells were injected into a single mammary fat pad of each mouse, and mice were euthanized 21 days after injection. All methods were done in accordance with and approved by The Institutional Animal Care and Use Committee at The Rockefeller University.

Preparation of tissue. All tissue was dissected and placed in $4 \%$ paraformaldehyde for fixed tissue samples. A test sample and an age-matched corresponding wild type (WT) sample were placed on the same slide for all experiments.

Immunofluorescence. The immunofluorescence detection of endogenous tissue antibody was performed at the Molecular Cytology Core Facility of Memorial SloanKettering Cancer Center using a Discovery XT processor (Ventana Medical Systems, Tucson AZ). Avidin Biotin block was applied for 12 minutes followed by incubation with biotinylated secondary antimouse IgG (Vector Labs, MOM Kit BMK-2202) in $1: 200$ dilution $(6.5 \mathrm{ug} / \mathrm{mL})$. Detection was performed with Blocker D and Streptavidin-HRP D (Ventana Medical Systems), followed by incubation with Tyramide-Alexa Fluor 488 (Invitrogen, cat \#T20992). Negative controls were prepared using biotinylated secondary anti-rabbit IgG (Vector Labs). IgM and IgA studies were processed as above (Invitrogen cat \#M31515, M31115). Slides for macrophage (CD-45) and myeloid (Iba-1) markers were blocked for 30 minutes with $10 \%$ normal rabbit serum, $2 \%$ BSA and $10 \%$ normal goat serum, $2 \%$ BSA, respectively. Primary antibodies rat anti-mouse CD45 (BD Pharmingen, cat \#550539, at $0.2 \mathrm{ug}$ / $\mathrm{ml}$ ) or rabbit anti-Iba-1 (Wako, cat \#019-19741, at $0.5 \mathrm{ug} / \mathrm{ml}$ ) were incubated for 3 hours, followed by incubation with either biotinylated rabbit anti-rat IgG (Vector, cat \# BA-4000) or biotinylated goat anti-rabbit IgG (Vector labs, cat\# : PK6101) in $1: 200$ dilution. Detection was performed as described above. All slides were counterstained with DAPI. No blockers for Fc receptor was used in any of the slides.

Image analysis. All slides were digitally scanned at the Molecular Cytology Core Facility of Memorial Sloan-Kettering Cancer Center using the Zeiss Mirax Scanner with $20 \times / 0.8 \mathrm{NA}$ objective. Exposure time depended on mouse model and antibody tested.

Pathology analysis. Adjacent sections were given to a veterinary histopathologist in the Center of Comparative Medicine and Pathology at the Laboratory of Comparative Pathology who was blinded to all sources of tissue and all immunofluorescence results. These adjacent section slides were stained with hematoxylin and eosin (H\&E) and all tissues were analyzed and graded. All histologically abnormal areas were marked and described by the degree of abnormality. In the liver histology sections, the following grading system, determined by the pathologist, was applied: $0=$ normal tissue, 1 = areas found to have other cellular changes such as karyomegaly, cytomegaly, cystoplasmic vacuolization, 2 = a focus of defined cellular alteration, $3=$ an adenoma causing compression of adjacent parenchyma, and $4=$ carcinoma.

Electron microscopy. Liver tissues from the Alb/c-myc model and C57Bl/6 model of mice were fixed in $4.0 \%$ paraformaldehyde and $0.1 \%$ glutaraldehyde in $0.1 \mathrm{M}$ sodium cacodylate buffer ( $\mathrm{pH}$ 7.4) overnight. Sections were processed for immunoelectron microscopy to recognize existing antibody as described previously ${ }^{27}$. Summation of tissue processing included quenching endogenous peroxidase with $0.5 \%$ hydrogen peroxide, blocking nonspecific antibody binding with $3 \%$ bovine serum albumin, applying biotinylated anti-mouse IgG, visualizing the immunocomplex with Vectastain ABC Kit (Vector Laboratories, Burlingame, CA) and peroxidase base reaction in the presence of $0.5 \% 3,3^{\prime}$ diaminobenzidine, and application of silver enhancement procedure to DAB immunoreactive products. Subsequently, sections were re-fixed with $2.5 \%$ glutaraldehyde in $0.1 \mathrm{M}$ sodium cacodylate buffer, dehydrated by a graded series of ethanol, postfixed with $1 \%$ osmium tetra-oxide and embedded in EMBed812. Ultra- thin sections were cut and examined in the electron microscope (100CX JEOL, Tokyo, Japan) with the digital imaging system (XR41-C, Advantage Microscopy Technology Corp, Danver, MA). All EM was done in the Electron Microscopy Resource Center at The Rockefeller University.

Statistical analysis. Statistical analysis was performed using GraphPad Prism 6 and Microsoft Excel. All intensity histogram values were normalized by tissue area. Analysis was performed on the top $60 \%$ of intensities. Groups were compared using the Mann-Whitney $U$ test. All $p$ values less than 0.05 were considered statistically significant. Means and ratios are presented with standard error of the mean.

\section{Results}

Alb/c-myc model. Overexpression of the c-myc oncogene is frequently seen in $\mathrm{HCC}^{28}$. We used a mouse model of HCC which overexpresses c-Myc under the control of an albumin promoter ${ }^{29}$. We probed with fluorescently tagged horse anti-mouse IgG for the presence of endogenous antibodies in formalin fixed, paraffin embedded liver tissue from this tumor model and from C57Bl6 wild-type (WT) mice. Similar results to experiments described below were observed with a goat anti-mouse IgG.

The anti-mouse IgG fluorescent signal, normalized for area of tumor, was approximately 28 -fold higher in all transgenic liver tissue from Alb/c-myc mice relative to tissue from WT mice $(0.04 \pm 6.1 \times$ $10^{-3}$ vs. $\left.1.4 \times 10^{-3} \pm 5.1 \times 10^{-4}\right)$ (Fig. 1a,b). The increased amount of mouse IgG in the Alb/c-myc tissue over the WT tissue was statistically significant ( $\mathrm{p}=0.0002 ; \mathrm{n}=8)$. However, the fluorescence of the anti-mouse IgG was heterogeneously distributed throughout the $\mathrm{Alb} / \mathrm{c}-m y c$ liver. Some areas demonstrated high levels of fluorescence, and therefore antibody content, while others were as dim as the liver from WT mice (Fig. 1a,c). Variation was noted both at the tissue and cellular level. The fluorescence was associated with sinusoidal endothelial cells and hepatocytes. Observations using confocal microscopy confirmed that the IgG was internalized in the hepatocytes as the IgG fluorescence was granular throughout the cell, but excluded from the nuclei (Fig. 1c). The binding of anti-mouse IgG to sinusoidal endothelial cells was confirmed with immuno-electron microscopy (Fig. 1d).

To test if the increased fluorescence of the anti-mouse IgG was the consequence of non-specific binding, samples were probed with a goat derived anti-rabbit IgG (supplemental figure 1a) or without an anti-IgG, (supplemental figure 1b). No signal was observed in Alb/c$m y c$ liver tissue with the anti-rabbit IgG or in the absence of anti-IgG. There was no appreciable difference between Alb/c-myc and WT tissue when probed with anti-mouse Immunoglobulin M (IgM) or anti-mouse Immunoglobulin A (IgA) (Supplementary Fig. 2a,b). These results indicate that the increased fluorescence using antimouse IgG was due to the specific recognition of mouse IgG within the tumor tissue.

To determine whether any of the anti-IgG fluorescence was associated with infiltrating immune cells, we stained for Iba-1 (macrophage marker), CD45 (marker of myeloid lineage) and CD45R (B cell maker) (Supplemental Fig. 3). As expected, these antibodies had distinct signals in splenic tissue. However, in Alb/c-myc liver sections processed in parallel on the same slide, we did not observe any IgG signal associated with B cells. These probes did not localize with the 
A.

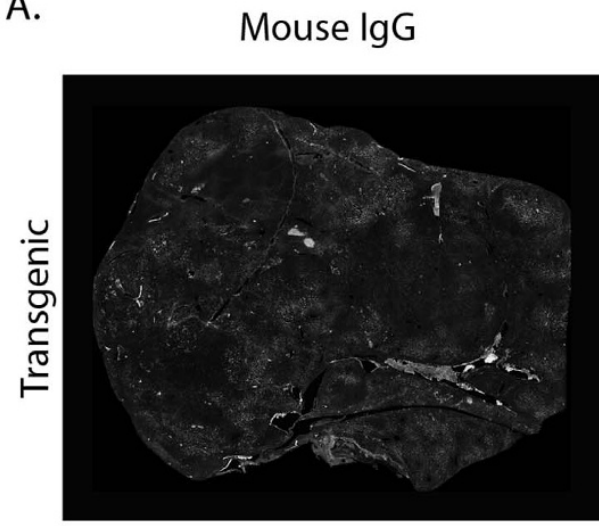

Mouse lgG (green) + DAPI (blue)
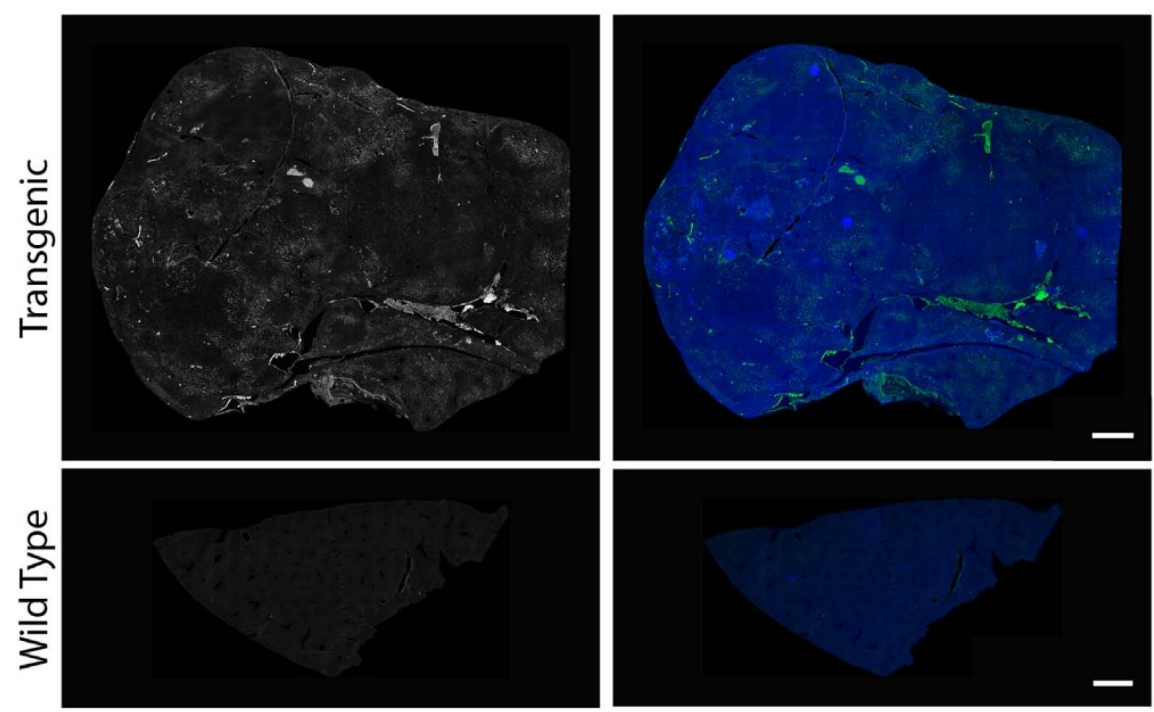

C.

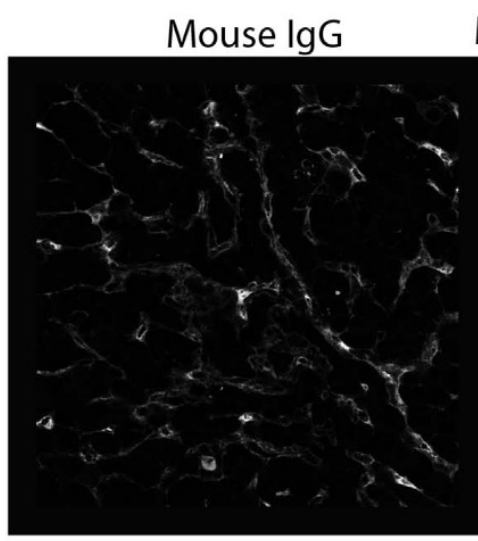

Mouse lgG (green) + DAPI (blue)
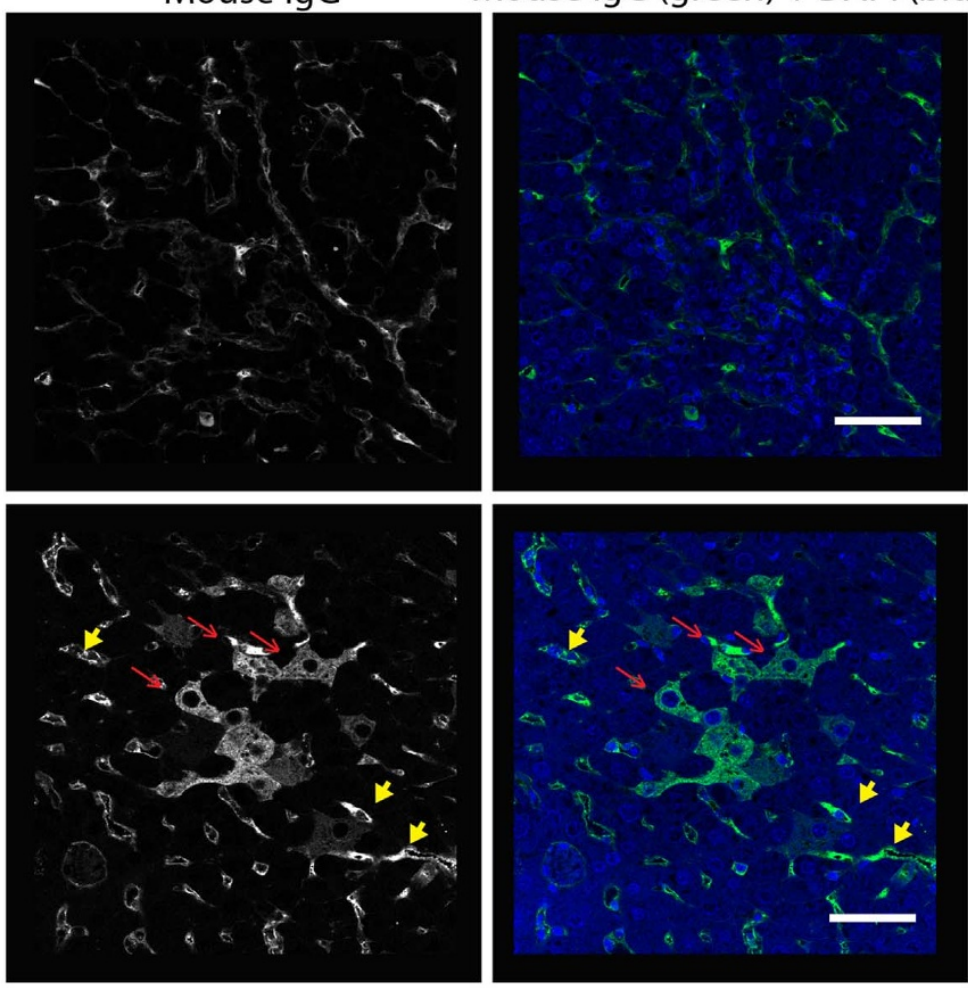

B.
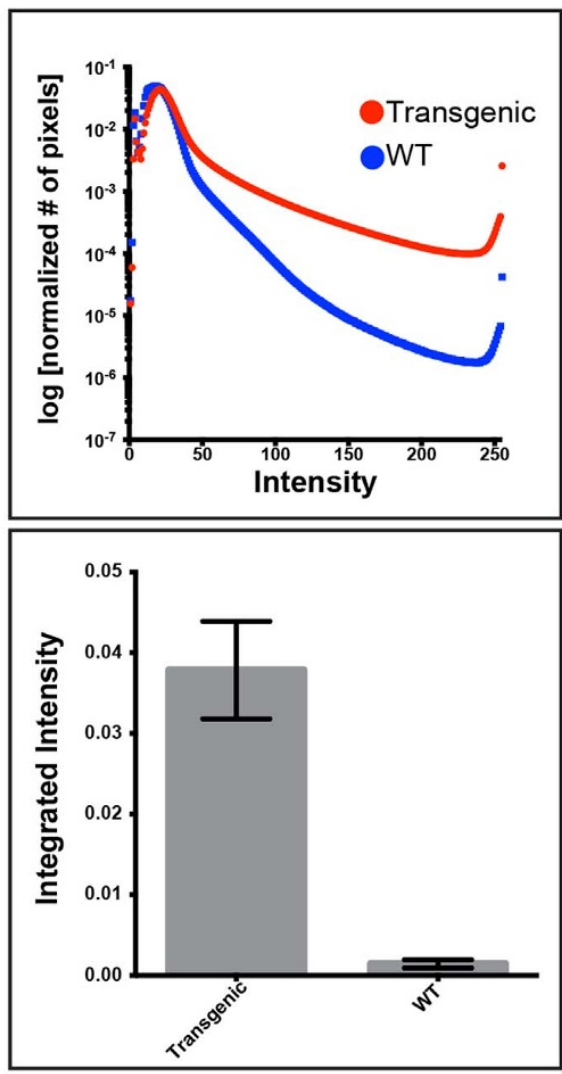

D.

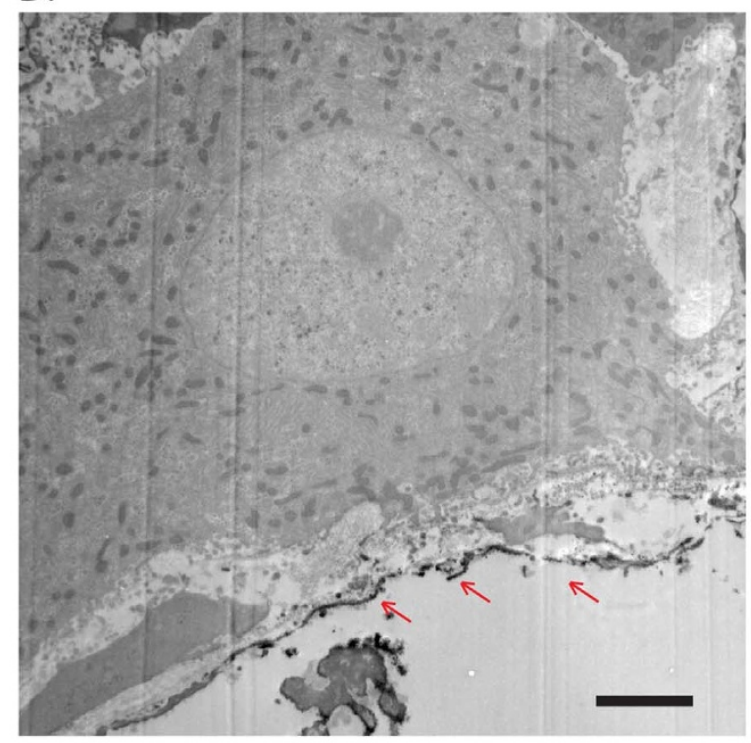

Figure $1 \mid$ In the alb-myc transgenic model of hepatocellular carcinoma, endogenous antibodies are identified at significantly higher levels than in matched wild-type controls. (A). Liver tissue stained with anti-mouse IgG labeled with AlexaFluor 488 viewed at low magnification demonstrates more antibody in tumor tissue than in normal wild type (WT) liver (scale bars $=1 \mathrm{~mm}$ ). (B). Integrated intensity along the top $60 \%$ of intensity values demonstrates 28 -fold greater fluorescence in tumor tissue than WT $(\mathrm{p}=0.0002)$. (C). Confocal slices through carcinoma tissue reveal two distinct staining patterns: staining of sinusoidal endothelial cells (yellow arrows); and staining of hepatocytes (red arrows) with the staining in the cytosol, but excluded from the nucleus, consistent with endocytosis of the antibody (scale bars $=50$ microns). (D). Binding to sinusoidal endothelial cells, arrows, was confirmed with immuno-electron microscopy (scale bar $=2 \mathrm{um}$ ).

overwhelming majority of anti-IgG fluorescence. These findings confirm that the increased signal from anti-mouse IgG was due to antibodies in the tumor, not to the presence of immune cells in the tissue.

Adjacent sections of liver samples were stained with $\mathrm{H} \& \mathrm{E}$ and analyzed by a histopathologist (data not shown). All Alb/c- myc tissue was graded as follows: 0 (normal), 1 (areas with cellular alterations), 2 (foci of alteration), 3 (adenoma causing compression) or 4 (carcinoma). All WT liver sections were confirmed to be histologically normal. Two Alb/c-myc mice had liver sections with grade 4 lesions, seven had grade 3 lesions, six had grade 2 lesions, and three had grade 1 lesions. The intensity of anti-mouse IgG fluorescence 
was significantly greater in all tumor grades relative to WT (grade 4: 64 -fold greater $(\mathrm{p}=0.04)$, grade 3 : 57 -fold greater $(\mathrm{p}=0.0003)$, grade 2 : 64 -fold greater $(\mathrm{p}=0.0007)$, grade $1: 58$-fold greater $(\mathrm{p}=$ $0.01)$, grade $0: 53$-fold greater $(\mathrm{p}=0.0002)$ ). When all abnormal grades were analyzed together (grades $1-4$ ), the fluorescent intensity was 62-fold greater than WT ( $\mathrm{p}<0.001)$ (Fig. 3a).

MMTV-neu model. Human mammary tumors contain amplification of HER-2/neu in $25-30 \%$ of patients ${ }^{24}$. We probed for the presence of endogenous antibodies in mouse mammary tissue from mice expressing the un-activated neu oncogene, driven by the mouse mammary tumor virus (MMTV) promoter $^{30}$, and from FVB WT mice. Mammary tissue was formalin fixed, paraffin embedded and probed with fluorescently labeled horse anti-mouse IgG; adjacent sections were stained with $H \& E(n=8)$. All tissue from the MMTV-neu mice had histological evidence of mammary carcinoma, and all WT mice were histologically normal, determined by a veterinary pathologist.

In MMTV-neu mammary tissue, the anti-mouse IgG had 26-fold greater fluorescent intensity than histopathologically normal WT mammary tissue $(p=0.001$, Fig. $2 a, c)$. The use of a goat anti-rabbit IgG produced no detectable signal in either MMTV-neu and WT tissue, establishing that the fluorescent signal is due to specific recognition of mouse IgG (supplemental figure 1a). There was no fluorescence detected in either tumor or WT tissue processed without antibody, eliminating autofluorescence as a source of the difference. Probing with anti-IgM and anti-IgA yielded no detectable difference between the MMTV-neu tissue and the WT tissue, consistent with the observations from the Alb/c-myc mice. This established mouse $\operatorname{IgG}$ as the predominant class of antibodies enriched within the tumor. In the abnormal mammary tissue, the mouse IgG was present on abnormal ductal and alveolar cells, the surrounding adipocytes, collagen, and skeletal muscle, as well as within glandular secretions (Fig. 2b). The detection of the anti-mouse IgG within the tumor was variable, with somewhat less signal observed in the center relative to the periphery of larger tumors (Fig. 2a).

Mouse models for prostate cancer. Myc is overexpressed in 30\% of human prostate cancers and expression in mice leads to prostatic intraepithelial neoplasia (PIN), followed by invasive adenocarcinoma. We tested for the presence of endogenous antibodies in the tissue of two different mouse models of prostate cancer. The first model expressed human Myc under the prostate-specific PB promoter ${ }^{31}$.

PTEN is deleted in $70-80 \%$ of human prostate cancers ${ }^{25,32}$ and prostate specific deletion also results in murine prostate cancer ${ }^{33}$. The second prostate tumor model was a knock-out of the PTEN tumor suppressor that does not progress to cancer but in a restricted number of cases does progress to a pre-neoplastic state.

PB-Myc mice had histologically evident prostate carcinoma (six of six mice) while all samples from wild-type mice were histologically normal. When probed with a fluorescently tagged horse derived antimouse IgG, PB- $M y c$ prostate tissue had 10.4-fold greater fluorescent intensity than respective WT tissue $(\mathrm{p}=0.002)$ (Fig. 4a,c). Again, there was no detected fluorescence when tissue was processed with goat-derived anti-rabbit IgG, eliminating the possibility of non-specific binding to the tumor. There was no difference between the two tissues when processed without any antibody, eliminating the contribution autofluorescence. Also, when probed with anti-mouse IgM or anti-mouse IgA, there was no difference between tumor and WT tissue, establishing the predominance of IgG as the class of mouse antibodies bound to the abnormal tissue.

In PTEN mice, all prostate samples (six of six mice) had histologically evident prostatic intraepithelial carcinoma and all WT samples were histologically normal. Unlike the previous models, there was considerable heterogeneity in anti-mouse IgG binding throughout the prostate and within PIN lesions (Fig. 4b) with fluorescence throughout the tumor microenvironment, including the stroma and fibrovascular structures surrounding abnormal regions, as well as prostatic secretions.

The fluorescence of three of the six PTEN prostate tissues was 11fold, 4-fold, and 8- fold greater than the WT prostate tissue. However, in the other three mice, the fluorescence of the WT was 3-fold, 16-fold, and 1.4-fold greater than the PTEN prostate tissue. This illustrates the heterogeneity of the IgG binding in this particular model system, consistent with the reported heterogeneity of the penetrance of the $P T E N+/-$ phenotype. As a result of the variability, the fluorescence intensity of the tumor samples $\left(7.18 \times 10^{-3} \pm 2.67\right.$ $\times 10^{-3}$ ) was only two fold-greater than the respective WT tissue $\left(3.42 \times 10^{-3} \pm 1.06 \times 10^{-3}, \mathrm{p}=0.57\right)$. The variability of the fluorescence within the PTEN model may explain the lack of statistical significance. There was no signal detected when tissue was processed with anti-rabbit IgG, or any difference in signal without any antibody, with anti-mouse IgM or anti-mouse IgA.

Probing of organs in MMTV-neu model. In the MMTV-neu model, we tested if there was an increased level of endogenous IgG in tissues other than the mammary tissue as compared to WT. While the MMTV promoter is predominantly expressed in mammary tissue, it has also been reported to be partially expressed in lung, salivary gland, and spleen, but at a 500 -fold lower level ${ }^{26}$.

All organs, other than MMTV-neu mammary tissue, were found to be histologically normal. The fluorescence signal from anti-IgG antibodies showed no statistical difference between MMTV-neu and wild-type in endogenous antibody in the liver $(\mathrm{p}=0.33)$, colon $(\mathrm{p}=$ $0.66)$, spleen $(\mathrm{p}>0.99)$, stomach $(\mathrm{p}>0.99)$, lung $(\mathrm{p}=0.33)$, brain ( $\mathrm{p}$ $=0.33)$, heart $(\mathrm{p}=0.33)$ and kidney $(\mathrm{p}=0.33)$ compared to WT. Comparing the ratio of the intensity of MMTV-neu tissue to WT tissue, the mammary tissue had the greatest intensity of antibody binding compared to all other organs (Fig. 3b). The next brightest tissue was the lung, which is known to have MMTV expression, and the MMTV-neu model is known to metastasize to the lung, although metastatic foci were not identified on histologic review.

Xenograft model. To determine the immune response to malignant cells in the background of a WT host without a transgene, we also tested a xenograft mouse model of breast cancer. The $4 \mathrm{~T} 1$ cell line was injected into a single mammary fat pad in four C57Bl6 mice, and mice were dissected on day 21 post-injection. The intensity of fluorescence in the tumor tissue was 10 -fold greater than in the WT mammary tissue ( $p=0.03$, Fig. $5 a, b)$. When this cell line was injected into SCID immunocompromised mice (CBySmn.CB17$\left.\operatorname{Prkd} c^{s c i d} / \mathrm{J}\right)$ unable to produce mature B cells, there was no antimouse IgG identified (Supplementary Fig. 4). Comparable to all other models, there was no signal in anti-rabbit IgG binding between the tumor and WT mammary tissue, and no difference detected without antibody, IgM, and IgA.

In all but one mouse, the remaining organs, including the contralateral mammary tissue, were histopathologically normal and binding of an anti-mouse IgG antibody was at low levels. In the remaining mouse, the liver, spleen, and lung had diffuse regions of increased antibody binding compared to organs from a WT mouse (Fig. 6). Upon histologic review of these three organs, an atypical myeloid hyperplasia/myeloid leukemia was identified (data not shown). The liver also contained an area of necrosis secondary to tumor infiltration. The other organs tested in this mouse (contralateral mammary tissue, skin, kidney, stomach, and colon) did not have any areas of increased endogenous antibody and were noted to be normal in histology.

\section{Discussion}

These results demonstrate that endogenous antibodies localize to neoplastic tissue in transgenic and xenograft mouse models of cancer. These antibodies are seen throughout the neoplastic tissue, 
A. Mouse lgG
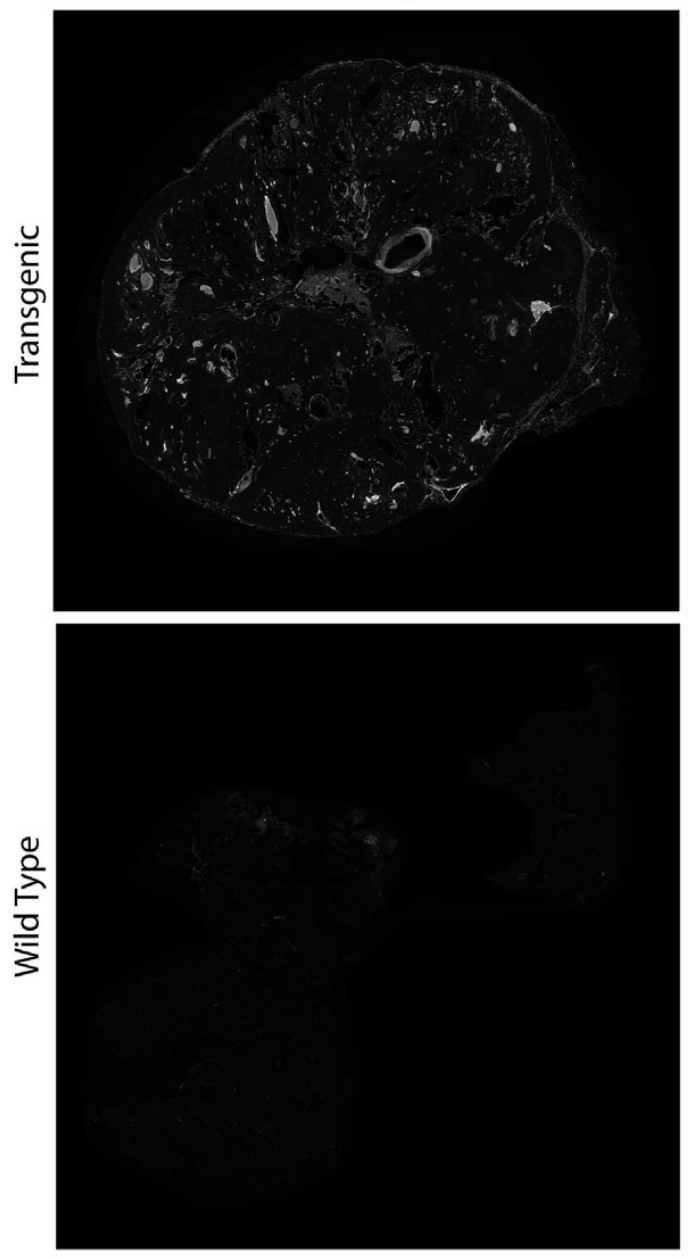

B. Mouse IgG

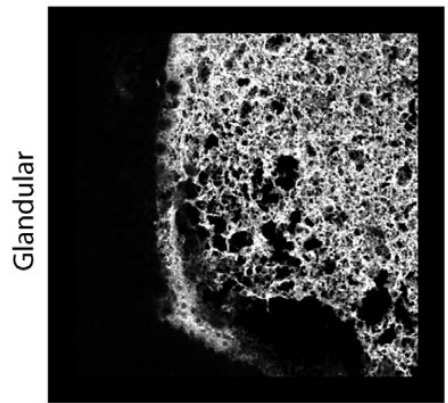

Mouse IgG (green) + DAPI (blue)

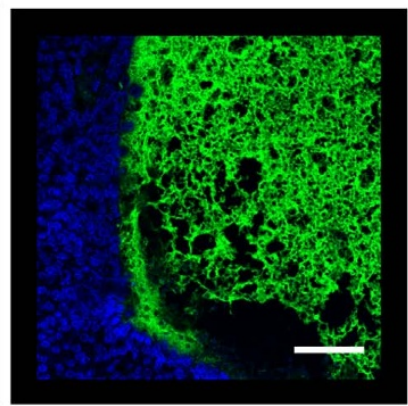

C.
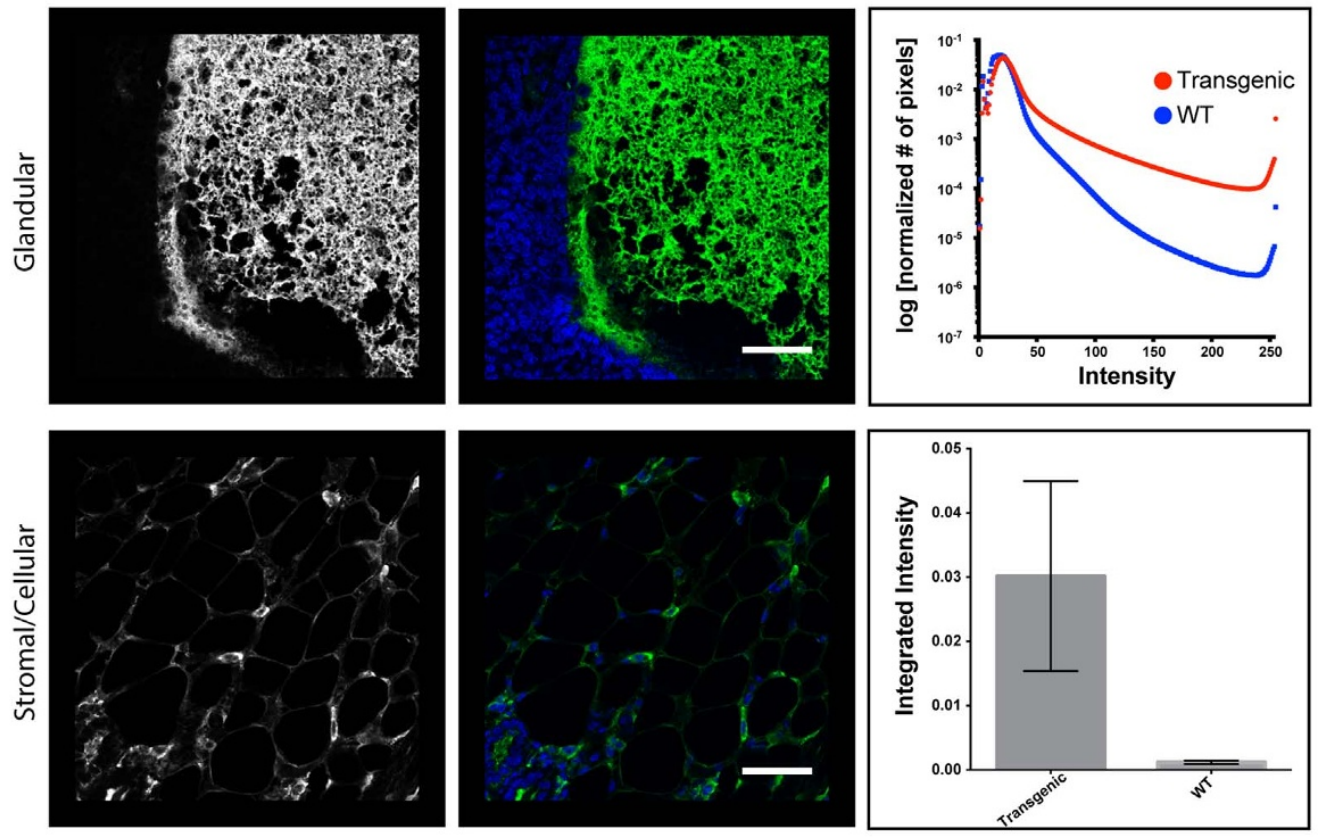

Figure 2 $\mid$ MMTV-neu is a transgenic model of murine mammary cancer. (A). Staining for endogenous antibody within the neoplastic tissue demonstrated increased overall fluorescence compared to wild type mammary tissue (scale bar $=1 \mathrm{~mm}$ ). (B). On confocal microscopy, the fluorescent signal corresponded to intraluminal glandular regions as well as the stroma (scale bar $=50 \mathrm{um}$ ). (C). Fluorescence labeling of mouse IgG in neoplastic mammary tissue is statistically higher than in wild type mammary tissue $(\mathrm{p}=0.001)$. 
A.

Alb-myc Neoplasia Grades

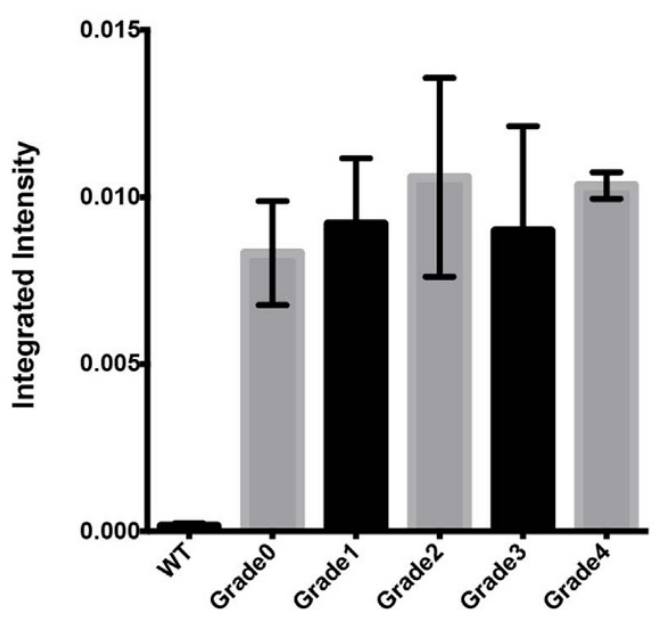

B.

\section{Ratio of MMTV to WT organs}

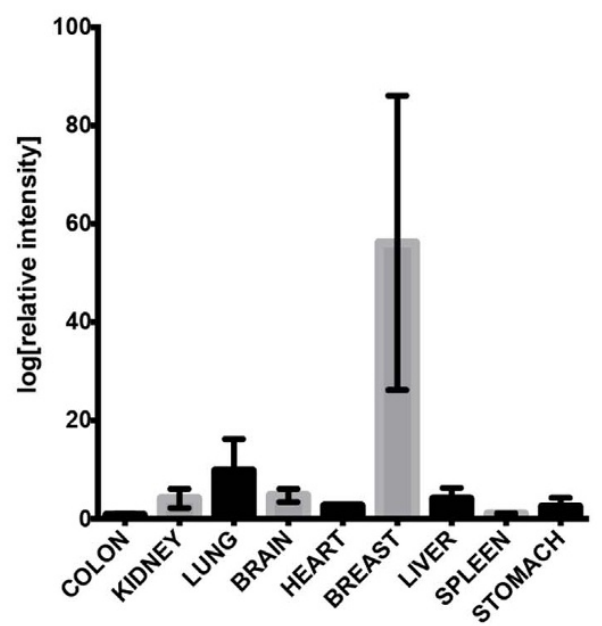

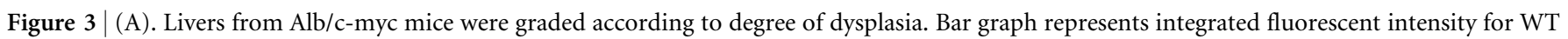

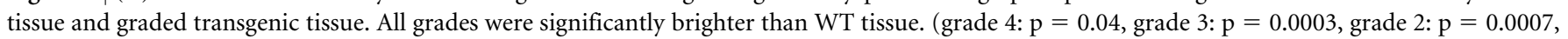
grade $1: \mathrm{p}=0.01$, grade $0: \mathrm{p}=0.0002)$. (B). Organs from transgenic MMTV-neu mice were stained with anti-mouse antibody to determine the

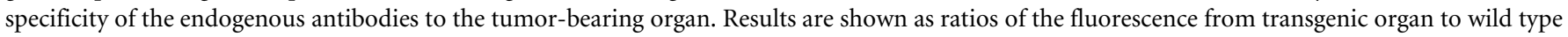
organ for colon, kidney, lung, brain, heart, breast, liver, spleen, and stomach.

involving numerous different types of cells, but they are not identified in corresponding tissue of WT controls. Since the cells of a tumor are heterogeneous in genotype and phenotype, we believe that the endogenous IgG represents a diverse collection of antibodies bound to a variety of epitopes.

In our analysis, we compared the fluorescent intensity throughout the organ, rather than only within the highly fluorescent regions. The heterogeneity of the immune response throughout the tumor tissue increased the variance of the cumulative fluorescence signal. This resulted in underestimation of the effect size, yet the results remained statistically significant.

Endogenous antibodies produced against an individual's own malignant cells provide the potential advantages of increased sensitivity and robustness for detecting and targeting pre-neoplastic and neoplastic lesions. This is the consequence of a myriad of unique features of antibodies. First, they have diverse epitope specificity and recognize a broad variety of antigens. Second, they undergo affinity maturation, improving the specificity and strength with which they bind. Furthermore, the antibody repertoire is continuously adapting to mutations that occur in the tumor over time. These features offer the potential to recognize the diversity of transformed cells within a tumor, as well as the ability to continue to detect these cells as they evolve.

The next critical step is to explore these observations in human tumors. If endogenous antibodies can detect neoplastic lesions, using a probe specific for the constant region of IgG would provide means for the continuous detection of existing neoplastic lesions, the identification of remote abnormal foci, and the assessment of resection margins for transformed cells. Additionally, this technology has the potential sensitivity to detect neoplasia at the level of the single immunoreactive cell as well as the expression of isolated aberrant immunogenic proteins. With endogenous antibodies continually tracking tumors, a probe developed to bind IgG minimizes mechanisms for tumor escape. If one epitope is altered or down regulated, the immune system will continue to recognize other antigens present on the neoplastic cells.

Our results suggest that early transitioning cells can be distinguished from surrounding tissue. First, grades 1-4 in the Alb/c-myc model all have significantly more antibody than the corresponding
WT tissue. Grade 1 lesions display changes at the nuclear and cellular level, and endogenous antibodies recognize these cells undergoing early changes. Second, portions of grade 0 transgenic tissue have antibody present, possibly localizing to very early lesions, not distinguishable on H\&E staining. Lastly, the antibodies recognized a subset of lesions in the PTEN model, which does not form frankly cancerous lesions but produces precancerous PIN. Thus, the antibodies may be recognizing epitopes present on the surface of these cells that reflect early biochemical changes within the cells. This may be helpful in detection of pre-neoplastic regions.

Endogenous antibodies have been observed within human tumors, including papillary thyroid cancer, melanoma and some soft tissue sarcomas ${ }^{22,23}$. Our findings confirm these data in various mouse models of cancer. This demonstrates the robustness of this finding, raising the potential of using IgG as a biomarker for distinguishing altered from normal tissue. Some studies have investigated the apparent local expression of IgG by tumor cells in breast cancer and various soft tissue tumors, including rhabdomyosarcoma and leiomyosarcoma ${ }^{22}$. However, we did not see an increase in IgG signal in tumors from SCID mice, whose B and T cells are functionally deficient. This suggests that the robustness of the detected signal in the immunocompetent models was derived from a systemic, host response, not local production.

The tumor has been described as an ecosystem: neoplastic cells with a milieu that interacts and aids in their growth ${ }^{34}$. In all of our models tested, endogenous antibodies were identified within the tumor microenvironment. The microenvironment is known to be made up of cells including fibroblasts, dendritic cells, leukocytes, and endothelial cells, that are activated or recruited by the nearby tumor cells to aid in the sustainment and growth of the tumor itself ${ }^{35}$. The communication between this reactive microenvironment and the cancer cells affects the phenotype of the tumor and disrupting this hospitable microenvironment has a detrimental effect on tumors ${ }^{36,37}$. Our results show that endogenous antibodies are also present in the microenvironment. In the Alb/c-myc model, antibodies appear to bind to the hepatocytes, but also to the sinusoidal endothelial cells. Alterations in these endothelial cells have been reported in early stages of liver tumorigenesis ${ }^{38}$, and they are involved with tumor survival, proliferation, and invasiveness ${ }^{39}$ In the MMTV-neu model, 
A.

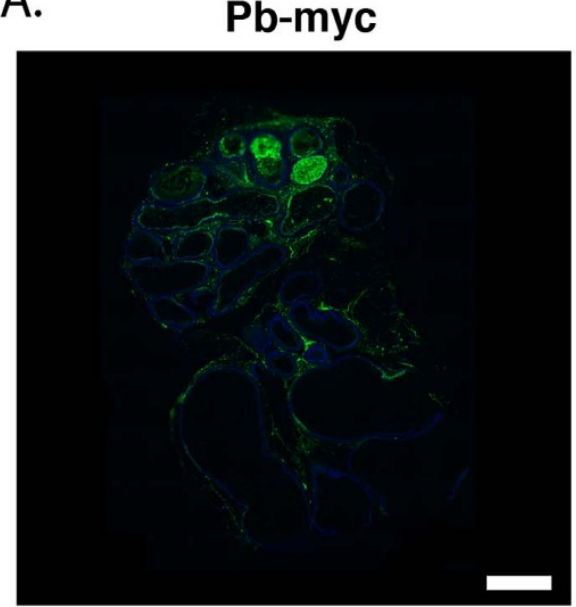

B.
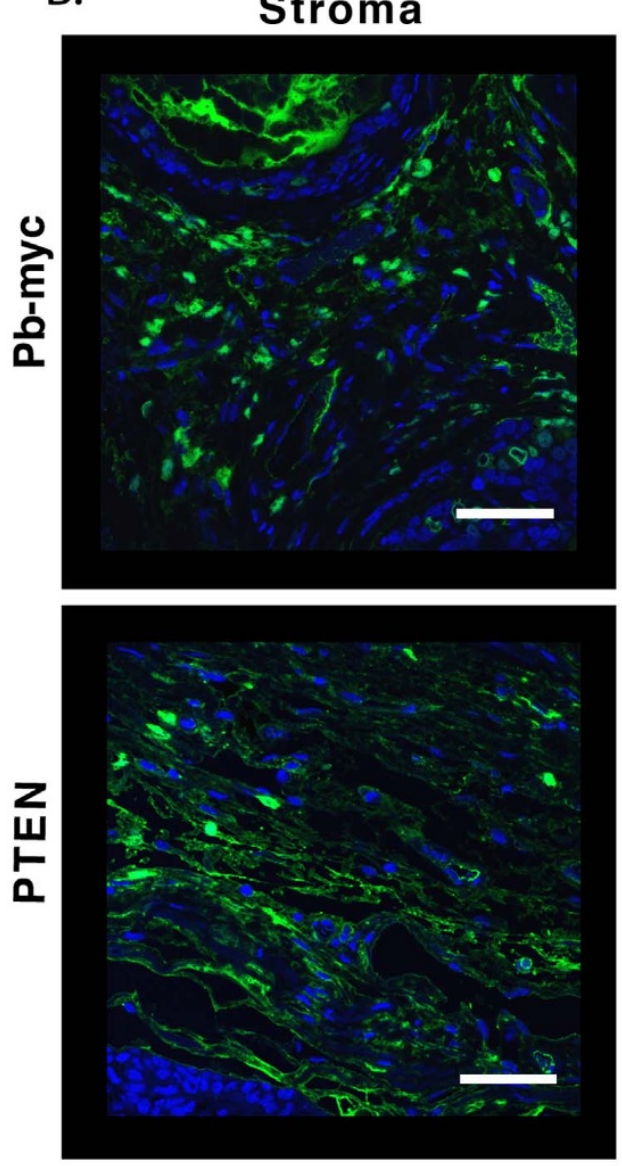

PTEN
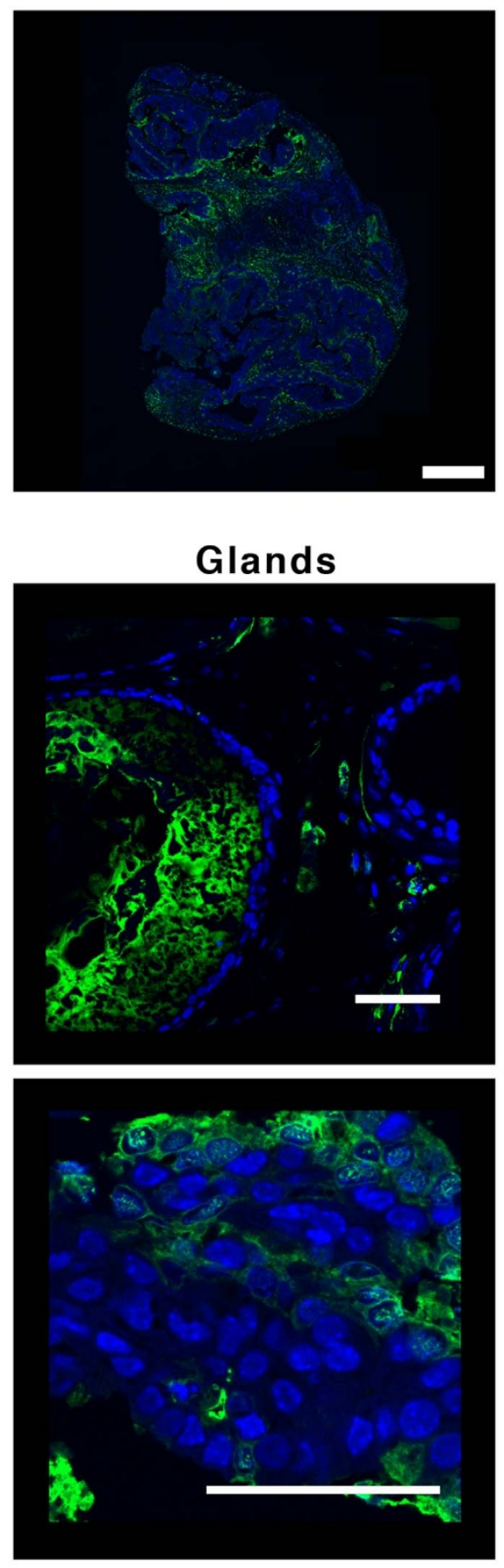

Wild Type

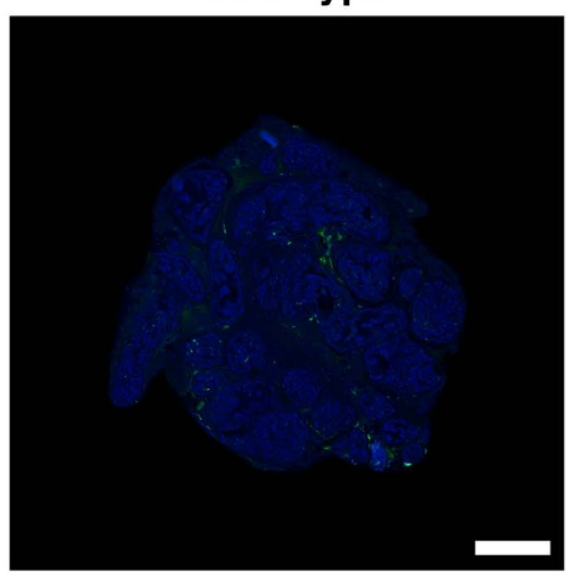

C.
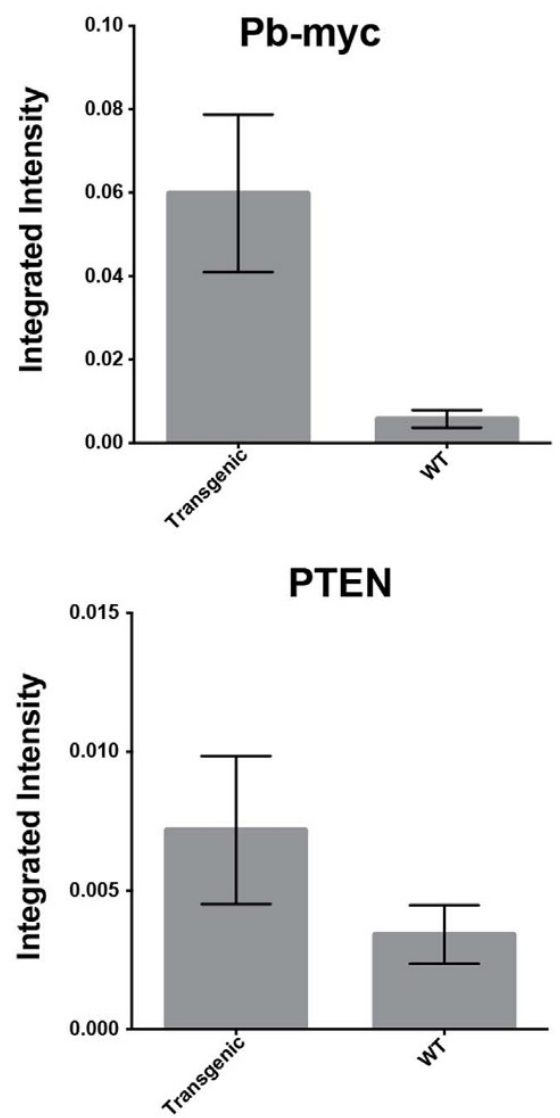

Figure $4 \mid$ (A). In two transgenic models of prostate cancer, more endogenous antibody was identified in tumor bearing tissue than wild type when viewed at low magnification. (B). High magnification confocal images demonstrate antibody localization. In the transgenic Pb-myc model, antibody localized to stroma and intraglandular regions within the neoplastic tissue. In mice with prostate-specific knockout of PTEN, antibody was localized to prostate stroma as well as glandular epithelial cells. (C). The difference in fluorescent intensity between malignant prostates and wild type prostates was statistically significant in the carcinoma-bearing $\mathrm{Pb}-\mathrm{myc}(\mathrm{p}=0.002)$ model but not in the preneoplastic PTEN model.

antibody is bound to the mammary tissue adipocytes, endothelial cells and connective tissue. Stroma adjacent to breast tumor undergoes both phenotypic and epigenetic transformations during tumorigenesis $^{40-42}$. Furthermore, breast cancer-associated fibroblasts have been shown to promote tumor growth ${ }^{43,44}$. Similar results were observed in the transgenic prostate models, with antibody bound throughout the microenvironment, which has been demonstrated to support the growth of the adjacent prostate tumor ${ }^{45,46}$.
Antibody fluorescence was slightly less intense within central regions of a tumor mass. This could be the result of endogenous antibodies having decreased accessibility to the central regions of the tumor or decreased binding. Alterations in the size and leakiness of a tumor's blood vessels could lead to ineffective blood flow and increased interstitial pressure within the tumor. Additionally, lymphatic vessels within tumors are altered and may contribute to intratumoral hypertension. These factors could impede the ingress of 
A.
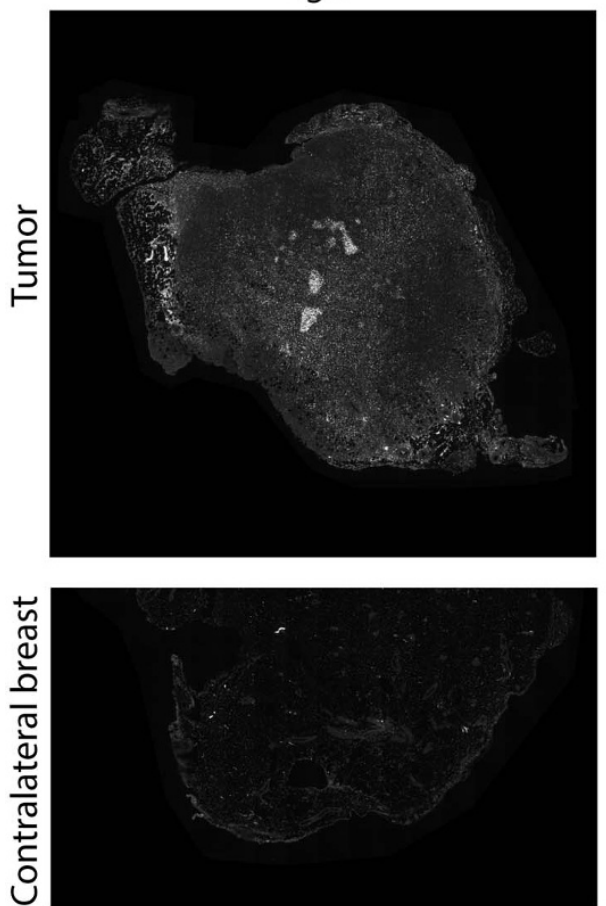

$\operatorname{lgG}($ green) + DAPI (blue)
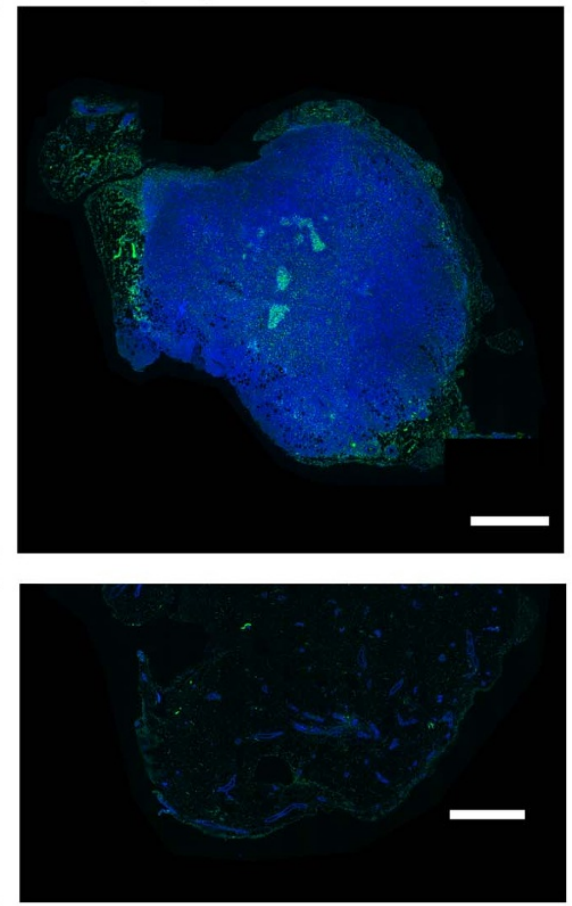

B.

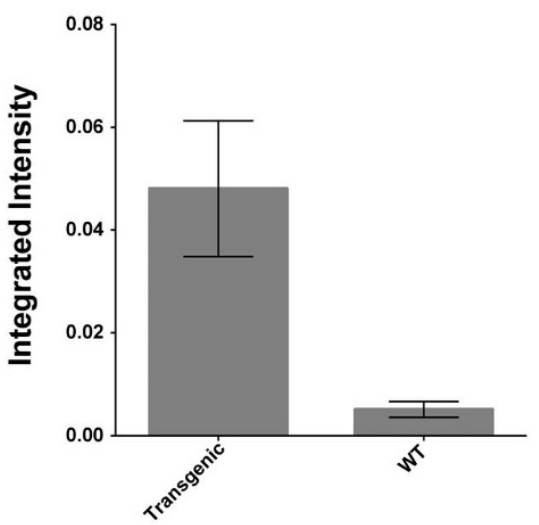

Figure $5 \mid$ In a 4T1 xenograft model of murine mammary cancer, tumors were implanted into the mammary fat pad of Balb/c mice. (A). The tumor bearing tissue contained more antibody localizing to both the malignant cells as well as the adjacent mammary tissue when compared to the contralateral non-injected mammary tissue (scale bar $=1 \mathrm{~mm}$ ). (B). The difference in fluorescence when the tissue was stained was statistically significant $(\mathrm{p}=0.03)$.

antibodies to the central regions of the tumor ${ }^{47}$. Furthermore, high affinity binding sites for the endogenous antibodies on the periphery of the tumor may restrict infiltration and binding to the central region of a tumor ${ }^{48,49}$.
Our results indicate that the presence of endogenous antibody in a tumor is the result of a tumor-specific immune response. First, antimouse IgG was the predominant immunoglobulin present in all models tested. This demonstrates both that the signal is specifically
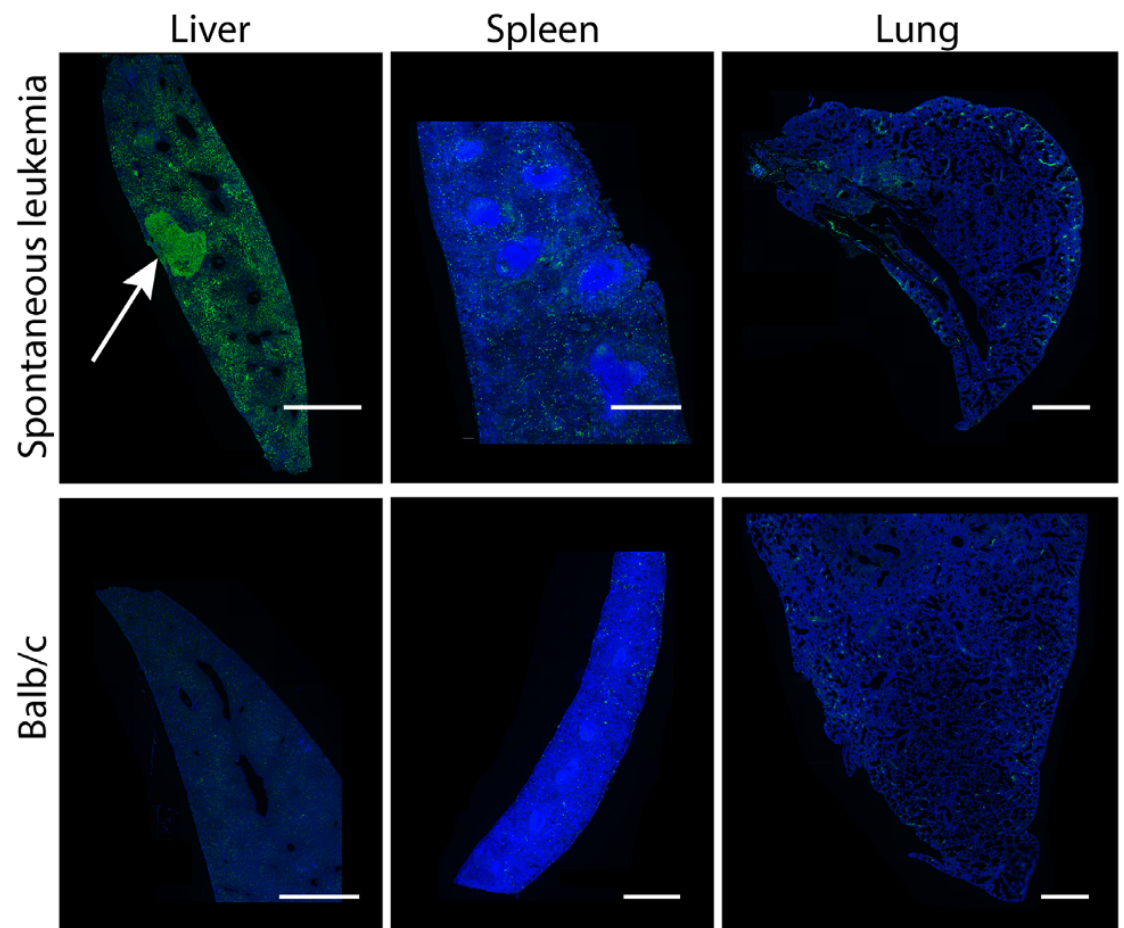

Figure 6 Sections from a Balb/c mouse with spontaneous AMH/leukemia in the liver, spleen, and lung. Tissue sections were stained with anti-mouse IgG conjugated to Alexa Fluor 488 and DAPI. Increased fluorescence is noted in tumor-bearing organs compared to corresponding wild type Balb/c mouse. The liver containing spontaneous leukemia (top left) contained a region of tumor necrosis (arrow). Scale bar $=500 \mu \mathrm{m}$. 
from the endogenous antibodies, not from nonspecific binding of the probe, and that the signal is from mouse IgG. Second, the increased presence of endogenous IgG was seen both in a xenograft model and in transgenic models, which systemically express a promoter/transgene. Third, the IgG was enriched in the tumor bearing organs. For example, when comparing antibody binding across organs within the MMTV-neu mice, the mammary gland has the highest relative intensity compared to the corresponding WT tissue. In the xenograft model, the antibody response was specific to where the tumor was grafted.

Significantly, in the xenografted mouse with spontaneous acute myeloid hyperplasia/leukemia, increased levels of antibody were detected in the liver, spleen, and lung, localizing to the same regions identified as pathologic on H\&E analysis. Thus, the results from the genetically engineered and xenograft solid tumor models are consistent with the observations from a spontaneous cancer. A spontaneous murine tumor is potentially a better model for the underlying biology of the human immune response to malignancy. Although limited to a single observation, this finding of antibody binding in a spontaneous tumor further suggests that the signal demonstrated in other experiments is not simply the effect of a transgene or xenograft.

Although neoplastic tissues and the microenvironment contain IgG, the precise host response remains to be defined. Further study will elucidate which epitopes are being bound, why there is a heterogeneous binding pattern, whether there is a physiologic significance to this binding, and why binding of IgG fails to result in elimination of the tumor cells. Future work will address the mechanisms, targets, and significance of these tissue-bound antibodies.

Focusing on endogenous tissue antibodies, independent of the target antigens, allowed us to distinguish neoplastic lesions from normal tissue in three transgenic and one xenograft model of cancer. Further investigation into a similar response in human cancers may allow the use of endogenous tissue antibodies to improve upon the ability of current imaging modalities to detect both pre-neoplastic and neoplastic tissue. This will lead to improved staging of disease, identification of occult metastasis, and earlier diagnosis of tumor recurrence. Furthermore, the ability to target the endogenous antibodies enriched in the tumor environment offers a potential therapeutic modality for eradicating pre-neoplastic and neoplastic tissue.

1. Gerlinger, M. et al. Intratumor heterogeneity and branched evolution revealed by multiregion sequencing. N. Engl. J. Med. 366, 883-892 (2012).

2. Cancer Genome Atlas Network, Comprehensive molecular portraits of human breast tumours. Nature 490, 61-70 (2012).

3. Loeb, L. A., Loeb, K. R. \& Anderson, J. P. Multiple mutations and cancer. Proc. Natl. Acad. Sci. U.S.A. 100, 776-781 (2003).

4. Shipitsin, M. et al. Molecular definition of breast tumor heterogeneity. Cancer Cell 11, 259-273 (2007)

5. Wilting, R. H. \& Dannenberg, J.-H. Epigenetic mechanisms in tumorigenesis, tumor cell heterogeneity and drug resistance. Drug Resist. Updat. 15, 21-38 (2012).

6. Gupta, P. B. et al. Stochastic state transitions give rise to phenotypic equilibrium in populations of cancer cells. Cell 146, 633-644 (2011).

7. Willimsky, G. \& Blankenstein, T. The adaptive immune response to sporadic cancer. Immunol. Rev. 220, 102-112 (2007).

8. Birkeland, S. A. et al. Cancer risk after renal transplantation in the Nordic countries, 1964-1986. Int. J. Cancer 60, 183-189 (1995).

9. Penn, I. Sarcomas in organ allograft recipients. Transplantation 60, 1485-1491 (1995).

10. Penn, I. Malignant melanoma in organ allograft recipients. Transplantation 61, 274-278 (1996).

11. Pham, S. M. et al. Solid tumors after heart transplantation: lethality of lung cancer. Ann. Thorac. Surg. 60, 1623-1626 (1995).

12. Dunn, G. P., Bruce, A. T., Ikeda, H., Old, L. J. \& Schreiber, R. D. Cancer immunoediting: from immunosurveillance to tumor escape. Nat. Immunol. 3 , 991-998 (2002).

13. Adams, G. P. \& Weiner, L. M. Monoclonal antibody therapy of cancer. Nat. Biotechnol. 23, 1147-1157 (2005).

14. Akcakanat, A. et al. NY-ESO-1 expression and its serum immunoreactivity in esophageal cancer. Cancer Chemother. Pharmacol. 54, 95-100 (2004).

15. Stockert, E. et al. A survey of the humoral immune response of cancer patients to a panel of human tumor antigens. J. Exp. Med. 187, 1349-1354 (1998).
16. Chapman, C. J. et al. Autoantibodies in lung cancer: possibilities for early detection and subsequent cure. Thorax 63, 228-233 (2008).

17. Korangy, F. et al. Spontaneous tumor-specific humoral and cellular immune responses to NY-ESO-1 in hepatocellular carcinoma. Clin. Cancer Res. 10, 4332-4341 (2004).

18. Maio, M. et al. Analysis of cancer/testis antigens in sporadic medullary thyroid carcinoma: expression and humoral response to NY-ESO-1. J. Clin. Endocrinol. Metab. 88, 748-754 (2003).

19. Fosså, A. et al. NY-ESO-1 protein expression and humoral immune responses in prostate cancer. Prostate 59, 440-447 (2004).

20. Zhang, J.-Y. \& Tan, E. M. Autoantibodies to tumor-associated antigens as diagnostic biomarkers in hepatocellular carcinoma and other solid tumors. Expert Rev. Mol. Diagn. 10, 321-328 (2010).

21. Kobold, S., Lütkens, T., Cao, Y., Bokemeyer, C. \& Atanackovic, D. Autoantibodies against tumor-related antigens: incidence and biologic significance. Hum. Immunol. 71, 643-651 (2010).

22. Chen, Z. et al. Immunoglobulin $\mathrm{G}$ is present in a wide variety of soft tissue tumors and correlates well with proliferation markers and tumor grades. Cancer 116 1953-1963 (2010).

23. Qiu, Y. et al. Immunoglobulin G expression and its colocalization with complement proteins in papillary thyroid cancer. Mod. Pathol. 25, 36-45 (2012).

24. Slamon, D. J. et al. Studies of the HER-2/neu proto-oncogene in human breast and ovarian cancer. Science 244, 707-712 (1989).

25. Gray, I. C. et al. Mutation and expression analysis of the putative prostate tumoursuppressor gene PTEN. Br. J. Cancer 78, 1296-1300 (1998).

26. Henrard, D. \& Ross, S. R. Endogenous mouse mammary tumor virus is expressed in several organs in addition to the lactating mammary gland. J. Virol. 62, 3046-3049 (1988).

27. Uryu, K., MacKenzie, L. \& Chesselet, M. F. Ultrastructural evidence for differential axonal sprouting in the striatum after thermocoagulatory and aspiration lesions of the cerebral cortex in adult rats. Neuroscience 105, 307-316 (2001).

28. Gu, J. R., Hu, L. F., Cheng, Y. C. \& Wan, D. F. Oncogenes in human primary hepatic cancer. J Cell Physiol Suppl 4, 13-20 (1986).

29. Murakami, H. et al. Transgenic mouse model for synergistic effects of nuclear oncogenes and growth factors in tumorigenesis: interaction of c-myc and transforming growth factor alpha in hepatic oncogenesis. Cancer Res. $\mathbf{5 3}$ $1719-1723$ (1993).

30. Guy, C. T. et al. Expression of the neu protooncogene in the mammary epithelium of transgenic mice induces metastatic disease. Proc. Natl. Acad. Sci. U.S.A. 89, 10578-10582 (1992).

31. Ellwood-Yen, K. et al. Myc-driven murine prostate cancer shares molecular features with human prostate tumors. Cancer Cell 4, 223-238 (2003).

32. Whang, Y. E. et al. Inactivation of the tumor suppressor PTEN/MMAC1 in advanced human prostate cancer through loss of expression. Proc. Natl. Acad. Sci. U.S.A. 95, 5246-5250 (1998).

33. Trotman, L. C. et al. Pten dose dictates cancer progression in the prostate. PLoS Biol. 1, E59 (2003).

34. Bissell, M. J. \& Radisky, D. Putting tumours in context. Nat. Rev. Cancer 1, 46-54 (2001).

35. Coussens, L. M. \& Werb, Z. Inflammation and cancer. Nature 420, 860-867 (2002).

36. Mueller, M. M. \& Fusenig, N. E. Friends or foes - bipolar effects of the tumour stroma in cancer. Nat. Rev. Cancer 4, 839-849 (2004).

37. Roskelley, C. D. \& Bissell, M. J. The dominance of the microenvironment in breast and ovarian cancer. Semin. Cancer Biol. 12, 97-104 (2002).

38. Frachon, S. et al. Endothelial cell marker expression in dysplastic lesions of the liver: an immunohistochemical study. J. Hepatol. 34, 850-857 (2001).

39. Franses, J. W., Baker, A. B., Chitalia, V. C. \& Edelman, E. R. Stromal endothelial cells directly influence cancer progression. Sci Transl Med 3, 66ra5 (2011).

40. Fiegl, H. et al. Breast cancer DNA methylation profiles in cancer cells and tumor stroma: association with HER-2/neu status in primary breast cancer. Cancer Res. 66, 29-33 (2006).

41. Hu, M. et al. Distinct epigenetic changes in the stromal cells of breast cancers. Nat. Genet. 37, 899-905 (2005).

42. Trimboli, A. J. et al. Pten in stromal fibroblasts suppresses mammary epithelial tumours. Nature 461, 1084-1091 (2009).

43. Orimo, A. et al. Stromal fibroblasts present in invasive human breast carcinomas promote tumor growth and angiogenesis through elevated SDF-1/CXCL12 secretion. Cell 121, 335-348 (2005).

44. Tyan, S.-W. et al. Breast cancer cells induce cancer-associated fibroblasts to secrete hepatocyte growth factor to enhance breast tumorigenesis. PLOS ONE 6, e15313 (2011).

45. Dakhova, O. et al. Global gene expression analysis of reactive stroma in prostate cancer. Clin. Cancer Res. 15, 3979-3989 (2009).

46. Chung, L. W. K., Baseman, A., Assikis, V. \& Zhau, H. E. Molecular insights into prostate cancer progression: the missing link of tumor microenvironment. J. Urol. $173,10-20$ (2005).

47. Jain, R. K. Physiological barriers to delivery of monoclonal antibodies and other macromolecules in tumors. Cancer Res. 50, 814s-819s (1990).

48. Fujimori, K., Covell, D. G., Fletcher, J. E. \& Weinstein, J. N. A modeling analysis of monoclonal antibody percolation through tumors: a binding-site barrier. J. Nucl. Med. 31, 1191-1198 (1990). 
49. Weinstein, J. N. \& van Osdol, W. The macroscopic and microscopic pharmacology of monoclonal antibodies. Int. J. Immunopharmacol. 14, 457-463 (1992).

\section{Acknowledgments}

We thank Katia Manova-Todorova and the staff at the Molecular Cytology core facility at Memorial Sloan-Kettering Cancer Center for helpful suggestions and guidance. We thank Can Pham and Herman Stellar for the Alb/c-myc mouse model and Brett Carver, Yu Chen, and Charles Sawyers for both mouse models of prostate cancer. Funding was provided by the Fibrolamellar Foundation, DeLiver A Cure, The Sohn Foundation. Supported in part by grant \# 8 UL1 TR000043 from the National Center for Advancing Translational Sciences (NCATS), National Institutes of Health (NIH) Clinical and Translational Science Award (CTSA) program to The Rockefeller University Center for Clinical and Translational Science subaward to BSR, JNH and SMS.

\section{Author contributions}

"B.R. participated in research design, in the performance of the research, data collection, data analysis, and writing the manuscript. J.H. participated in research design, in the performance of the research, data collection, and writing the manuscript. D.D. participated in research design, in the performance of the research, data collection, and writing the manuscript. P.S. participated in research design, in the performance of the research, data collection, and writing the manuscript. A.W. participated in research design, in the performance of the research, data collection, and editing manuscript. I.P.L. participated in research design, in the performance of the research, data collection, and editing manuscript. L.J. participated in histopathological analysis, and editing manuscript. M.G. participated in statistical analysis, and editing the manuscript. J.S. participated in conceiving the experiments, research design, and writing the manuscript. M.L. participated in research design, and editing the manuscript. S.S. participated in conceiving the experiments, research design, data analysis and writing."

\section{Additional information}

Supplementary information accompanies this paper at http://www.nature.com/ scientificreports

Competing financial interests: The authors declare no competing financial interests. How to cite this article: Rich, B.S. et al. Endogenous Antibodies for Tumor Detection. Sci. Rep. 4, 5088; DOI:10.1038/srep05088 (2014).

This work is licensed under a Creative Commons Attribution-NonCommercialNoDerivs 3.0 Unported License. The images in this article are included in the article's Creative Commons license, unless indicated otherwise in the image credit; if the image is not included under the Creative Commons license, users will need to obtain permission from the license holder in order to reproduce the image. To view a copy of this license, visit http://creativecommons.org/licenses/by-nc-nd/3.0/ 